\title{
SUBGROUPS OF FREE SOLVABLE GROUPS
}

\author{
JACQUES LEWIN* and TEKLA LEWIN* \\ (Received 13 May 1969, revised 10 September 1969) \\ To Bernhard Hermann Neumann on his 60th birthday \\ Communicated by G. E. Wall
}

\section{Introduction}

A consequence of Schreier's formula is that if $G$ is a subgroup of the free group $F$ of rank $n>1$ and rank $G \leqq n$, then $G=F$ or $G$ is of infinite index in $F$. However, if $S$ is a free solvable group of derived length $l>1$ and $H$ is a subgroup of $S$ which is free solvable of the same length, then the rank of $H$ does not exceed the rank of $S$. These observations led G. Baumslag to conjecture that if $H$ is of finite index in $S$ then $H=S$. In fact, we have sharper results in two directions. If $H$ and $S$ are free solvable of the same length, not only is $H$ of infinite index in $S$, but $\delta_{l-1}(S) / \delta_{l-1}(H)$ is torsion-free. In another direction we need not assume that $S$ is free solvable, only that $S$ is torsion-free and of derived length $l(l>1)$ and that $H$ is not cyclic. Thus Stallings' theorem [11] that a finitely generated torsionfree group with a free subgroup of finite index is itself free has an even stronger counterpart in the variety of groups solvable of length at most $l(l>1)$ : a torsionfree group in that variety with a non-cyclic free subgroup of finite index coincides with this subgroup. The proof relies on the following theorem: If $S$ is a free solvable group, $J$ is the group of automorphisms of $S$ which induce the identity on $S / S^{\prime}$, and $I$ is the group of inner automorphisms of $S$, then $J / I$ is torsion-free. The proofs of these theorems form the bulk of the first four sections.

Another consequence of the Schreier technique is that a normal subgroup of a free group of finite rank is either infinitely generated or of finite index. By analogy one is led to conjecture that if $S$ and $H$ are free solvable of derived length $l>1$, $S$ not cyclic, then $H$ is normal in $S$ implies $H=S$. This is correct, but much more is true: If $H$ is not cyclic, then $H$ is its own normalizer. If $S$ is free metabelian $(l=2)$ of finite rank, then another class of self-normalizing subgroups are those subgroups $K$ such that $K S^{\prime}=S$; we do not know whether, for $l>2$ and $S$ of finite rank, $K \delta_{l-1}(S)=S$ implies $K$ is its own normalizer. However, we have the following generalization. Let $G$ be a non-abelian solvable group such that all but possibly

* This work was supported by NSF Grant Number GP 8094. 
the last term $\delta_{l-1}(G)$ of the derived series of $G$ are finitely generated; if $K$ is a subgroup of $G$ such that $K \delta_{l-1}(G)=G$, then $K$ coincides with its normalizer.

If $H$ is a non-cyclic free solvable group of derived length $l$ which is normal in a group $G$ solvable of the same derived length, then we obtain as an immediate consequence of a theorem of Smel'kin that $\delta_{t-1}(G)=\delta_{t-1}(H) \times K$ where $K$ centralizes $H$.

Finally, in section 6 we have a rather easy proof of a fact that seems to have escaped notice: If $G$ is a finitely generated abelian by polycyclic group, so that $G$ has an abelian normal subgroup $N$ with $G / N$ polycyclic, and $K$ is a subgroup of $G$ such that $K N$ is of finite index in $G$, then $K$ is finitely generated: In particular, if $G$ is a finitely generated metabelian group and $K G^{\prime}$ is of finite index in $G$, then $K$ is finitely generated. (See [3] for a related result.)

The basic tool is the Magnus representation for free metabelian groups; the results we have for free solvable groups are proved by induction from the metabelian case.

\section{Notation and the Magnus representation}

In this section we collect some facts and notation which will be used throughout this paper.

We denote by $M$ the free metabelian group with basis $x_{1}, \cdots, x_{n}$. We set $A=M / M^{\prime}$ and $a_{i}=x_{i} M^{\prime}$. We denote by $T$ the free module with basis $t_{1}, \cdots, t_{n}$ over the integral group ring $\mathbb{Z} A$. Let $G$ be the group of matrices of the form $\left(\begin{array}{ll}a & 0 \\ t & 1\end{array}\right)$ with $a \in A$ and $t \in T ; G$ is the standard wreath product of a free abelian group (generated by $\left.\left(\begin{array}{ll}1 & 0 \\ t_{i} & 0\end{array}\right), i=1, \cdots, n\right)$ by a free abelian group $(A)$. The map

$$
x_{i} \rightarrow\left(\begin{array}{cc}
a_{i} & 0 \\
t_{i} & 1
\end{array}\right)
$$

defines a faithful representation $\mu$, the Magnus representation [7] of $M$ in $G$ specified by the basis $x_{1}, \cdots, x_{n}$. (As will be seen shortly, the dependence of $\mu$ on this basis is only apparent.) We will identify $M$ with its image in $G$. Thus $M^{\prime}$ is a subgroup of the group of matrices of the form $\left(\begin{array}{ll}1 & 0 \\ t & 1\end{array}\right)$, and we will identify the group of matrices of this form with $T$, via the identification $t=\left(\begin{array}{ll}1 & 0 \\ t & 1\end{array}\right)$.

If, for $x \in M$ and $g \in M^{\prime}$, we define $\left(x M^{\prime}\right) g=x^{-1} g x$, then $M^{\prime}$ is a $\mathbb{Z} A$ module, and this action of $\mathbb{Z} A$ on $M^{\prime}$ agrees with the action of $\mathbb{Z} A$ that $M^{\prime}$ inherits as a subset of $T . M^{\prime}$ is not a free $\mathbb{Z} A$-module for $n>2$, but the Magnus representation embeds $M^{\prime}$ in the free module $T$.

Let $x_{1}^{\prime}, \cdots, x_{k}^{\prime}$ be elements of $M$ with

$$
x_{i}^{\prime} \mu=\left(\begin{array}{cc}
a_{i}^{\prime} & 0 \\
t_{i}^{\prime} & 1
\end{array}\right) \text {. }
$$


If an element $\left(\begin{array}{ll}a & 0 \\ t & 1\end{array}\right)$ is in $g p\left(x_{1}^{\prime}, \cdots, x_{k}^{\prime}\right)$, then $a \in g p\left(a_{1}^{\prime}, \cdots, a_{k}^{\prime}\right)$ and $t \in \bmod \left(t_{1}^{\prime}, \cdots, t_{k}^{\prime}\right)$. Now suppose that $x_{1}^{\prime}, \cdots, x_{n}^{\prime}$ is another basis of $M$. Since $x_{i}=\left(\begin{array}{ll}a_{i} & 0 \\ t_{i} & 1\end{array}\right) \in g p\left(x_{1}^{\prime}, \cdots, x_{n}^{\prime}\right)$ for all $i, g p\left(a_{1}^{\prime}, \cdots, a_{n}^{\prime}\right)=A$ and $\bmod \left(t_{1}^{\prime}, \cdots, t_{n}^{\prime}\right)=T$. Thus the upper left-hand entries of a set of free generators of $M$ freely generate $A$, and the lower left-hand entries freely generate $T$. Hence two Magnus representationsof $M$ in $G$ are equivalent; that is, they differ only by an automorphism of $G$.

If $v_{1}, \cdots, v_{n}$ are elements of $\mathbb{Z} A$ or elements of $T$, we denote by $v_{r}$ and $v_{c}$ respectively the row and column vectors whose $i$-th coordinate is $v_{i}$. In particular $a_{c}$ denotes the column vector whose coordinates are $a_{1}, \cdots, a_{n}$, the generators of $A . \mathbf{1}_{c}$ and $\mathbf{1}_{r}$ denote the vectors whose coordinates are all 1.

The following theorem characterizes the embedding of $M$ in $G$.

THEOREM A. (S. Bachmuth [1]) The element $x=\left(\begin{array}{ll}a & 0 \\ \Sigma \alpha_{j} t_{3} & 1\end{array}\right)$ is in $M$ if and only if $\boldsymbol{\alpha}_{r}\left(\boldsymbol{a}_{c}-\mathbf{1}_{c}\right)=a-1$.

(If $x$ is in $M$, then $a$ turns out to be $x M^{\prime}$ and, it may be of some intercst to note, $\alpha_{j}$ is the partial derivative of $x$ with respect to $x_{j}$ taken in $\mathbb{Z} A$.)

We will be concerned mostly with free (metabelian) subgroups of $M$. The following theorem is fundamental.

TheOREm B. (G. Baumslag [2]) The elements $z_{1}, \cdots, z_{k}, k>1$, in a free solvable group $S$ of derived length l freely generate a free solvable subgroup of derived length $l$ if and only if $z_{1} S^{\prime}, \cdots, z_{k} S^{\prime}$ freely generate a free abelian group.

Suppose now that $L$ is a non-cyclic free subgroup of $M$. Then, by Theorem B, we may embed $L$ in a free subgroup $H$ of $M$ which, if the generators of $H$ and $M$ were suitably chosen, is freely generated by elements $y_{1}, \cdots, y_{n}$ with $y_{i}=x_{i}^{k_{i}}$ $\bmod M^{\prime}$ (each $k_{i}$ a non-zero integer) in such a way that $L$ is generated by $y_{1}, \cdots$, $y_{k}$. Then

$$
y_{i} \mu=\left(\begin{array}{cc}
a_{i}^{k_{i}} & 0 \\
u_{i} & 1
\end{array}\right) \quad i=1,2, \cdots, n
$$

with $u_{i} \in T$. Suppose that $u_{i}=\sum_{j} \gamma_{i j} t_{j}\left(\gamma_{i j} \in \mathbb{Z} A\right)$ and let $\Gamma$ be the matrix $\left(\gamma_{i j}\right)$. Let $B=H / H^{\prime}$. Since $H^{\prime}$ is a maximal abelian subgroup of $H, H \cap M^{\prime}=H^{\prime}$, and so $B$ can be identified with the subgroup of $A$ generated by $b_{1}=a_{1}^{k_{1}}, \cdots, b_{n}=a_{n}^{k_{n}}$.

Let $\mathfrak{a}$ be the augmentation ideal of $A$. Let $\mathfrak{b}$ be the augmentation ideal of $B$, i.e., the ideal of $\mathbb{Z} A$ generated by the elements $1-b$ with $b \in B$. We use bars to denote images under the natural $\operatorname{map} \mathbb{Z} A \rightarrow \mathbb{Z}(A / B)$ and under the maps it induces on the vectors and matrices with entries in $\mathbb{Z} A$.

LEMMA 1.

$$
\overline{\operatorname{det} \Gamma}=\sum_{g \in A / B} g
$$

Proof. Since, by Theorem A, $\Gamma\left(\boldsymbol{a}_{c}-\mathbf{1}_{c}\right)=b_{c}-\mathbf{1}_{c}$, we have $\bar{\Gamma}\left(\overrightarrow{\boldsymbol{a}_{c}-\mathbf{1}_{c}}\right)=0$. Thus if $\overline{\Gamma^{*}}$ is the adjoint of $\bar{\Gamma}$, we find that 


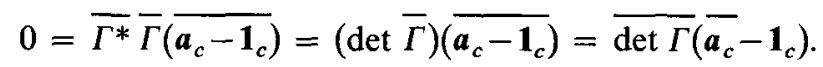

Therefore $\overline{\operatorname{det} \Gamma}\left(\bar{a}_{i}-1\right)=0$ for all $i$. That is, $\overline{\operatorname{det} \Gamma} \bar{a}_{i}=\overline{\operatorname{det} \Gamma}$ for all $i$, which implies that $\overline{\operatorname{det} \Gamma} g=\overline{\operatorname{det} \Gamma}$ for all $g \in A / B$. Suppose now that $\overline{\operatorname{det} \Gamma}=\sum_{h \in A / B} m_{h} h, m_{h}$ an integer. Then for all $g \in A / B$

$$
\sum_{h \in A / B} m_{h} h=\sum_{h \in A / B} m_{h} h g=\sum_{h \in A / B} m_{h g^{-1}} h .
$$

Thus $m_{h}=m_{h g^{-1}}$ for all $g$; that is, the coefficients are all the same and for some integer $m$

$$
\overrightarrow{\operatorname{det} \Gamma}=m \sum_{h \in A / B} h
$$
Since $\sum_{h \in A / B} h=\prod_{i}\left(1+\bar{a}_{i}+\cdots+\bar{a}_{i}^{k_{i}-1}\right)$ and the kernel of the bar map is $\mathfrak{b}$,
we have

$$
\operatorname{det} \Gamma=m \prod_{i}\left(1+a_{i}+\cdots+a_{i}^{k_{i}-1}\right) \bmod \mathfrak{b} \text {. }
$$

However, the fact that $y_{i}=x_{i}^{k_{i}} \bmod M^{\prime}$ implies that $u_{i}=\left(1+a_{\imath}+\cdots+a_{i}^{k_{i}-1}\right) t_{i}$ $\bmod a T$, and hence that

$$
\operatorname{det} \Gamma=\prod_{i}\left(1+a_{i}+\cdots+a_{i}^{k_{i}-1}\right) \bmod a .
$$

Therefore $m$ must be 1 .

Since in particular $\operatorname{det} \Gamma \neq 0$, the $u_{i}$ are $\mathbb{Z} A$-independent.

Recall that $L$ is generated by $y_{1}, \cdots, y_{k}$, where

$$
y_{i} \mu=\left(\begin{array}{ll}
b_{i} & 0 \\
u_{i} & 1
\end{array}\right) \quad i=1, \cdots, k \text {. }
$$

Let $C=g p\left(b_{1}, \cdots, b_{k}\right)$ and let $V$ be the $\mathbb{Z} C$-module generated by $u_{1}, \cdots, u_{k}$. Let $F$ be the subgroup of $G$ of matrices of the form $\left(\begin{array}{ll}c & 0 \\ v & 1\end{array}\right)$ with $c \in C$ and $v \in V$. Since the $u_{i}$ are $\mathbb{Z} A$-independent they are, a fortiori, $\mathbb{Z} C$-independent; therefore $V$ is a free $\mathbb{Z} C$-module with basis $u_{1}, \cdots, u_{k}$. This shows that if $\mu^{\prime}$ is the restriction of $\mu$ to $L$ cut down to $F$ then $\mu^{\prime}: L \rightarrow F$ is a Magnus representation of $L$. Now, $\mu$ is the Magnus representation of $M$ in $G$ specified by a carefully chosen set of free generators $x_{1}, \cdots, x_{n}$ of $M$, but as remarked earlier all the Magnus representations of $M$ in $G$ are equivalent, so that the previous statement is true for any Magnus representation $\mu$ of $M$. Thus we have shown

LEMMA 2. If $\mu: M \rightarrow G$ is a Magnus representation of the free metabelian group $M$ and $L$ is a non-cyclic free subgroup of $M$, then the restriction of $\mu$ to $L$ cut down to an appropriate subgroup $F$ of $G$ is a Magnus representation of $L$. (See above for the description of $F$.)

The interest in Lemma 2 is that it allows us to use Theorem $A$ to recognize elements of $L$. 
LEMMA 3. Let $K$ be a subgroup of $M$ for which $K M^{\prime}=M$, and let $V$ be the $\mathbb{Z} A$ module generated by the lower left-hand entries in the representations of elements of $K$. Let $x=\left(\begin{array}{ll}a & 0 \\ u & 1\end{array}\right)$ with $u \in V$. If $x \in M$, then $x \in K$.

Proof. Let $K=g p\left(x_{1} g_{1}, \cdots, x_{n} g_{n}, h_{1}, h_{2}, \cdots\right)$ where $g_{i}, h_{i} \in M^{\prime}$ and say

$$
x_{i} g_{i} \mu=\left(\begin{array}{ll}
a_{i} & 0 \\
u_{i} & 1
\end{array}\right), \quad h_{i} \mu=\left(\begin{array}{ll}
1 & 0 \\
h_{i} & 1
\end{array}\right) .
$$

Then $V=\bmod \left(u_{1}, \cdots, u_{n}, h_{1}, h_{2}, \cdots\right)$. Suppose that $u=\Sigma \rho_{i} u_{i}+\Sigma \sigma_{i} h_{i}, \rho_{i} \in \mathbb{Z} A$, $\sigma_{i} \in \mathbb{Z} A$, and that $x=\left(\begin{array}{ll}a & 0 \\ u & 1\end{array}\right)$ is an element of $M$. Then

$$
x=\left(\begin{array}{cc}
a & 0 \\
\Sigma \rho_{i} u_{i} & 1
\end{array}\right)\left(\begin{array}{cc}
1 & 0 \\
\Sigma \sigma_{i} h_{i} & 1
\end{array}\right) .
$$

Now, if $P$ is a subset of $M^{\prime}$, then $g p_{M}(P)$ is the $\mathbb{Z} A$-module generated by $P$. Since $K M^{\prime}=M, K \cap M^{\prime}$ is normal in $M$. Therefore $\left(\begin{array}{ll}1 & 0 \\ \Sigma \sigma_{i} h_{i} & 1\end{array}\right) \in K$. It follows that $\left(\begin{array}{cc}a & 0 \\ \Sigma \rho_{i} u_{i} & 1\end{array}\right) \in M$. Thus it is sufficient to prove the lemma in the case that $K$ is free, say $K=g p\left(x_{1} g_{1}, \cdots, x_{n} g_{n}\right)$. If $\Gamma$ is the matrix introduced above, then by Theorem A, $\rho_{r} \Gamma\left(a_{c}-\mathbf{1}_{c}\right)=a-1$ and $\Gamma\left(a_{c}-\mathbf{1}_{c}\right)=\left(a_{c}-\mathbf{1}_{c}\right)$; therefore $\rho_{r}\left(a_{c}-\mathbf{1}_{c}\right)$ $=a-1$. By Lemma 2, (1) provides a Magnus representation for $K$, and so by Theorem A, $\left(\begin{array}{cc}a & 0 \\ \Sigma_{\rho_{i} u_{i}} & 1\end{array}\right) \in K$ and the lemma is proved.

$S$ will denote a free solvable group of derived length $l$, usually of finite rank. If $G$ is a group, $\delta_{k}(G)$ and $\gamma_{k}(G)$ denote the $k$-th term of the derived and lower central series respectively. As usual we write $G^{\prime}$ for $\delta_{1}(G)$. If $X$ is a subset of $G$ we denote by $g p(X)$ and $g p_{G}(X)$ respectively the subgroup and normal subgroup generated by $X$.

\section{Free subgroups}

We can now prove our first theorem.

THeOREM 1. Let $S$ be a free solvable group of derived length $l>1$, and $L$ a free subgroup of $S$. Then $\delta_{l-1}(S) / \delta_{l-1}(L)$ is torsion-free.

Proof. If $L$ is cyclic, then $\delta_{l-1}(L)=1$. Let us assume that $L$ is not cyclic.

First, let $S=M$, a free metabelian group of finite rank $n$. We embed $L$ in a free subgroup $H$ of $M$ of rank $n$ as in section 1 and we use the notation developed there. We will show that $H^{\prime} / L^{\prime}, g p_{M}\left(H^{\prime}\right) / H^{\prime}$, and $M^{\prime} / g p_{M}\left(H^{\prime}\right)$ are all torsion-free, whence it follows that $M^{\prime} / L^{\prime}$ is.

Suppose that $y \in H$ and $y^{p}=z \in L$ for some integer $p$. Let $\pi$ be the projection of $H$ onto $L$ determined by $y_{i} \pi=y_{i}$ for $i=1, \cdots, k$ and $y_{i} \pi=1$ for $i=k+1$, $\cdots, n$. Then $(y \pi)^{p}=z$. However, $H$ is a group with unique roots (for it is easily calculated that $G$ is); hence $y=y \pi$, i.e., $y \in L$. Thus $H^{\prime} / L^{\prime}$ is torsion-free. 
Let $\left\{1=s_{1}, \cdots, s_{m}\right\}$ be a transversal for $B$ in $A$.

Let $U$ be the $\mathbb{Z} B$-module generated by $u_{1}, \cdots, u_{n}$ and $\bar{U}$ be the $\mathbb{Z} A$-module generated by $U$. Since $u_{1}, \cdots, u_{n}$ are $\mathbb{Z} A$-independent, $\bar{U}$ is a free $\mathbb{Z} A$-module freely generated by $u_{1}, \cdots, u_{n}$. Therefore, qua $\mathbb{Z} B$-modules,

$$
\bar{U}=\bigoplus_{i=1}^{m} s_{i} U .
$$

Now, $g p_{M}\left(H^{\prime}\right)$ is the $\mathbb{Z} A$-module generated by $H^{\prime}$, so $g p_{M}\left(H^{\prime}\right)=\sum_{a \in A} a H^{\prime}=$ $\sum_{i=1}^{m} s_{i} H^{\prime}$. Since $H^{\prime} \subset U$, it follows from (2) that this sum is direct:

$$
g p_{M}\left(H^{\prime}\right)=\bigoplus_{i=1}^{m} s_{i} H^{\prime}
$$

Therefore, since $H^{\prime}$ is torsion-free, $g p_{M}\left(H^{\prime}\right) / H^{\prime}$ is torsion-free.

We now wish to establish that $\bar{U} \cap M^{\prime}=g p_{M}\left(H^{\prime}\right)$. As we have seen,

$$
\Gamma\left(\boldsymbol{a}_{c}-\mathbf{1}_{c}\right)=b_{c}-\mathbf{1}_{c} .
$$

If $x \in \bar{U} \cap M^{\prime}$, then there is a row vector $\alpha_{r}=\left(\alpha_{1}, \cdots, \alpha_{n}\right)$ with $\alpha_{i} \in \mathbb{Z} A$ such that $x=\alpha_{r} u_{c}$. We write $\alpha_{r}=\sum_{i=1}^{m} s_{i} \alpha_{r}^{i}$, where $\alpha_{r}^{i}$ is a row vector with entries in $\mathbb{Z} B$. Now, $x=\alpha_{r} \Gamma t_{c}$ and by Theorem A

$$
\boldsymbol{\alpha}_{r} \Gamma\left(\boldsymbol{a}_{c}-\mathbf{1}_{c}\right)=0,
$$

since, for $x \in M^{\prime}, x=\left(\begin{array}{ll}1 & 0 \\ x & 1\end{array}\right)$. Thus,

$$
0=\alpha_{r}\left(b_{c}-\mathbf{1}_{c}\right)=\sum_{i=1}^{m} s_{i} \alpha_{r}^{i}\left(b_{c}-\mathbf{1}_{c}\right)
$$

Since $\alpha_{r}^{i}\left(\boldsymbol{b}_{c}-\mathbf{1}_{c}\right) \in \mathbb{Z} B, s_{i} \alpha_{r}^{i}\left(\boldsymbol{b}_{c}-\mathbf{1}_{c}\right)$ is a sum of group elements of $A$ which all lie in the coset $s_{i} B$ and hence we must have $\alpha_{r}^{i}\left(b_{c}-\mathbf{1}_{c}\right)=0$. By Lemma 2 and Theorem A the element $h_{i}=\alpha_{r}^{i} u_{c}$ is an element of $H^{\prime}$. Thus $x=\Sigma s_{i} h_{i}$ is in $g p_{M}\left(H^{\prime}\right)$. Hence $\bar{U} \cap M^{\prime} \subset g p_{M}\left(H^{\prime}\right)$. The reverse inclusion is obvious and $\bar{U} \cap M^{\prime}=$ $g p_{M}\left(H^{\prime}\right)$ as claimed.

It follows that

$$
M^{\prime} / g p_{M}\left(H^{\prime}\right)=M^{\prime} / \bar{U} \cap M^{\prime} \cong \frac{\bar{U}+M^{\prime}}{\bar{U}} .
$$

Thus $M^{\prime} / g p_{M}\left(H^{\prime}\right)$ is isomorphic to a submodule of $T / \bar{U}$, and we will now show that $T / \bar{U}$ has no elements of finite order. By a theorem of Fitting (see for example [6], theorem 6.15), since $T$ and $\bar{U}$ are free modules of the same rank, det $\Gamma$ annihilates $T / \bar{U}$.

Suppose now that $x \in T$ and that, for some prime integer $p, p x \in \bar{U}$. By the foregoing, $(\operatorname{det} \Gamma) x \in \bar{U}$ as well. Thus if 
and

$$
p x=\Sigma \beta_{i} u_{i} \quad \beta_{i} \in \mathbb{Z} A
$$

then we have

$$
(\operatorname{det} \Gamma) x=\Sigma \varepsilon_{i} u_{i} \quad \varepsilon_{i} \in \mathbb{Z} A,
$$

$$
\Sigma p \varepsilon_{i} u_{i}=\Sigma(\operatorname{det} \Gamma) \beta_{i} u_{i}
$$

Therefore $p \varepsilon_{i}=(\operatorname{det} \Gamma) \beta_{i}$ for all $i$. However, Lemma 1 says that $\overline{\operatorname{det} \Gamma}=\sum_{g \in A / B} g$, where $\overline{\operatorname{det} \Gamma}$ is the image of $\operatorname{det} \Gamma$ under the natural map $\mathbb{Z} A \rightarrow \mathbb{Z}(A / B)$. Since $p$ does not divide $\overline{\operatorname{det} \Gamma}, p$ does not divide det $\Gamma$. But $p$ is a prime in the unique factorization domain $\mathbb{Z} A$, so for each $i, \beta_{i}=p \beta_{i}^{\prime}$. Thus

$$
p x=p \Sigma \beta_{i}^{\prime} u_{i}
$$

Since $T$ is a torsion-free group, $x=\Sigma \beta_{i}^{\prime} u_{i}$; thus $x \in \bar{U}$.

We have now established that $M^{\prime} / L^{\prime}$ is torsion-free.

For the general case, it is first of all clear that if the theorem is true for finitely generated free metabelian groups, then it is true for all free metabelian groups. For if $L$ is a free subgroup of some free metabelian group $M$ and $x \in M^{\prime}$ with $x^{p} \in L^{\prime}$, then we can easily find finite subsets $y_{1}, \cdots, y_{i}$ and $x_{1}, \cdots, x_{j}$ of the free generators of $L$ and $M$ respectively such that $g p\left(y_{1}, \cdots, y_{i}\right) \subset g p\left(x_{1}, \cdots, x_{j}\right)$, $x \in g p\left(x_{1}, \cdots, x_{j}\right)$, and $x^{p} \in g p\left(y_{1}, \cdots, y_{i}\right)$.

For $S$ a free solvable group of derived length $l>2$ and $H$ a non-cyclic free subgroup of $S$ of length $l$, we establish the theorem by considering the free metabelian groups $\delta_{l-2}(S)$ and $\delta_{l-2}(H)$.

Notice that in the course of the proof of Theorem 1 we have established that if $H$ is a non-cyclic free metabelian subgroup of a free metabelian group of finite rank, then $g p_{M}\left(H^{\prime}\right)$ is the direct product of the distinct conjugates of $H^{\prime}$. For if we choose $z_{i}$ so that $z_{i} M^{\prime}=s_{i}$ for $i=1, \cdots, m$ (then $z_{1}, \cdots, z_{m}$ is a transversal for $H M^{\prime}$ in $\left.M\right)$, (3) says that $g p_{M}\left(H^{\prime}\right)=\chi_{i=1}^{m} H^{\prime z_{i}}$. The directness of this product shows these conjugates of $H^{\prime}$ are distinct, and since $H M^{\prime}$ normalizes $H^{\prime}$, these are all the conjugates. It follows that the normalizer of $H^{\prime}$ is $H M^{\prime}$. It is easily checked that the rank of $H$ being equal to the rank of $M$ does not enter into the argument.

For a free metabelian group $M$, if $L<M$ and $L^{\prime}=M^{\prime}$, then $L=M$. So by induction if $S$ is a free solvable group, $L<S$, and $\delta_{l-1}(L)=\delta_{l-1}(S)$, then $L=S$. Thus Theorem 1 establishes that proper free subgroups of free solvable groups are never of finite index, and Baumslag's conjecture is proved.

One might further conjecture that if $H$ is a free subgroup of the free metabelian group $M$, then the intersection of the conjugates of $H$ lies in $M^{\prime}$. This is false as the following example shows: Let $M$ be generated by $x_{1}$ and $x_{2}$ and let $H=$ $g p\left(x_{1}\left[x_{1}, x_{2}\right]^{x_{2}}, x_{2}\right)$. Then $H \neq M$, yet, as we shall show, the normal closure of $x_{2}^{6}$ lies in $H$. Since $H M^{\prime}=M$, then $H^{\prime}$ is normal in $M$ and $H^{\prime}=M^{\prime} \cap H$. Further, if $x \in H$, then $g p_{M}(x) \subset H$ if and only if $[x, M] \subset H$; thus $g p_{M}(x) \subset H$ if and 
only if $\left[x_{i}, x\right] \in H$ for $i=1,2$. Clearly $\left[x_{2}, x_{2}^{6}\right] \in H$. Now,

$$
\begin{aligned}
{\left[x_{2}, x_{1}\left[x_{1}, x_{2}\right]^{x_{2}}\right] } & =\left[x_{2}, x_{1}\right]\left[x_{2},\left[x_{1}, x_{2}\right]^{x_{2}}\right] \\
& =\left[x_{2}, x_{1}\right]\left(\left[x_{1}, x_{2}\right]^{x_{2}}\right)^{1-x_{2}}=\left[x_{2}, x_{1}\right]^{1+x_{2}\left(x_{2}-1\right)}
\end{aligned}
$$

is an element of $H$. Using the identity $x^{6}-1=(x-1)\left(x^{2}+x+1\right)(x+1)\left(x^{2}-x+1\right)$ we see that $1+x_{2}\left(x_{2}-1\right)$ divides the polynomial $x_{2}^{6}-1 / x_{2}-1=x_{2}^{5}+x_{2}^{4}+\cdots+1$. Thus

$$
\left[x_{2}^{6}, x_{1}\right]=\left[x_{2}, x_{1}\right]^{x_{2} 5+\cdots+1}=\left[x_{2}, x_{1}\right]^{\left(1+x_{2}\left(x_{2}-1\right)\right) p\left(x_{2}\right)}
$$

is an element of $H$, and we have proved our claim.

This example can be clarified by considering the converse situation. Suppose $H$ is a free subgroup of the non-cyclic free metabelian group $M$, and $N$ is the intersection of the conjugates of $H$. If $H$ is cyclic, then $N$ is a normal cyclic subgroup of $M$, and hence $N=1$. Otherwise, let $x \in N$. Then

$$
[x, M] \subset \bigcap_{z \in M}\left(M^{\prime} \cap H^{z}\right)=\bigcap_{z \in M} H^{\prime z} .
$$

But we have shown that the normalizer of $H^{\prime}$ is $H M^{\prime}$ and that the distinct conjugates of $H$ generate their direct product. It follows that unless $H M^{\prime}=M$ then $[x, M]=1$ and so $x=1$. Thus: If $M$ is a non-cyclic free metabelian group of finite rank and $H$ a free subgroup of $M$ with $H M^{\prime} \neq M$, then the intersection of the conjugates of $H$ is trivial. Suppose then that $H M^{\prime}=M$. Then $H^{\prime}$ is normal, so $H^{\prime}=H \cap M^{\prime}$ is contained in $N$. Now if $x \in H \backslash H^{\prime}, g p_{M}(x) \subset H$ if and only if $H^{\prime} \supset[x, M]=\left[x, H M^{\prime}\right]=[x, H]\left[x, M^{\prime}\right]$, which occurs if and only if $x$ centralizes $M^{\prime} / H^{\prime}$, that is to say, if and only if $x M^{\prime}-1$ annihilates the module $M^{\prime} / H^{\prime}$. Referring to the proof of Theorem 1 , since in the present case $H^{\prime}$ and $U$ coincide respectively with $g p_{M}\left(H^{\prime}\right)$ and $\bar{U}$, we find that

$$
M^{\prime} / H^{\prime} \cong \frac{M^{\prime}+U}{U} .
$$

However, $y_{i}=x_{i} \bmod M^{\prime}$ implies that $u_{i}=t_{i}+h_{i}$, where $h_{i} \in M^{\prime}$. Hence $M^{\prime}+U=T$, and $M^{\prime} / H^{\prime} \cong T / U$. So $g p_{M}(x) \subset H$ if and only if $x M^{\prime}-1$ annihilates $T / U$. If $M$ has rank $n$, the theorem of Fitting cited above gives the relation

$$
(\operatorname{ann}(T / U))^{n} \subset i d(\operatorname{det} \Gamma) \subset \operatorname{ann}(T / U) .
$$

Now, $x M^{\prime}$ is a power of some element in a suitably chosen basis for $A$, say $x M^{\prime}=a^{k}$. Thus if $g p_{M}(x) \subset H$, det $\Gamma$ divides $\left(a^{k}-1\right)^{n}$. Thus, up to units, all the prime divisors of det $\Gamma$ are polynomials in the single element $a$. It then follows that if $\alpha \in \operatorname{ann}(T / U)$, some prime divisor of $\alpha$ must be a polynomial in $a$. Thus we have the following curious fact: Let $M$ be a non-cyclic free metabelian group of finite rank and $H$ a free subgroup of $M$ such that $H M^{\prime}=M$. If $N$ is the intersection of the conjugates of $H$, then $N / N \cap M^{\prime}$ is cyclic (possibly trivial). Here $N \cap M^{\prime}=$ 
$H \cap M^{\prime}=H^{\prime}$. Further, the integer $k$ above cannot be a prime power for then none of the divisors of $a^{k}-1$ have coefficient sum 1, while det $\Gamma$ does.

\section{Automorphisms}

To prove the analogue of Stallings' theorem, we need some information about automorphism groups. The following theorem is due to Smel'kin [10].

THEOREM C. If $\eta$ is an automorphism of the free solvable group $S$ and $\eta$ fixes pointwise the last term of the derived series of $S$, then $\eta$ is an inner automorphism induced by an element of the last term of the derived series of $S$.

We also need the solvable analogue of a theorem of G. Baumslag and Tekla Taylor (Lewin) [4], proposition 1. The proof is closely related to the one in [4] and goes via two lemmas.

LEMMA 4. Let $M$ be a free metabelian group and let $x$ be an element of $M^{\prime}$ such that $x$ is a $k$-th power modulo every term of the lower central series of $M$. Then $x$ is a $k$-th power in $M$.

Proof. We may clearly assume that $M$ is finitely generated. Still in the notation of section 1 , let $T_{n}$ be the module $T / \mathfrak{a}^{n} T$, a free $\mathbb{Z} A / \mathfrak{a}^{n}$-module, and let $G_{n}$ be the group of matrices of the form $\left(\begin{array}{ll}a & 0 \\ t+a^{n} T & 1\end{array}\right)$, with $a \in A, t \in T$. If $\eta_{n}$ is the natural epimorphism of $T$ onto $T_{n}$, then the map $\bar{\eta}_{n}:\left(\begin{array}{ll}a & 0 \\ t & 1\end{array}\right) \rightarrow\left(\begin{array}{ll}a & 0 \\ t \eta_{n} & 1\end{array}\right)$ is clearly an epimorphism of $G$ onto the nilpotent group $G_{n}$. Hence $x \bar{\eta}_{n}$ has a $k$-th root for every $n$. Suppose $x=\left(\begin{array}{ll}1 & 0 \\ t & 1\end{array}\right)$; then for each $n$ we can find an element $b \in A$ and an element $s \in T_{n}$ such that

$$
\left(\begin{array}{cc}
1 & 0 \\
t \eta_{n} & 1
\end{array}\right)=\left(\begin{array}{ll}
b & 0 \\
s & 1
\end{array}\right)^{k}=\left(\begin{array}{cc}
b^{k} & 0 \\
\left(1+b+\cdots+b^{k-1}\right) s & 1
\end{array}\right) .
$$

It follows that $b=1$ and $t \eta_{n}=k s$. Thus, if $t=\Sigma \alpha_{i} t_{i}$ it follows that each $\alpha_{i}$ is divisible by $k$ modulo every $\mathfrak{a}^{n}$. Thus $\alpha_{i} \in \bigcap_{n=1}^{\infty}\left(k \mathbb{Z} A+\mathfrak{a}^{n}\right)$. However, a theorem in the theory of noetherian rings states that for any ideal $I$ of $\mathbb{Z} A, \bigcap_{n=1}^{\infty}\left(I+\mathfrak{a}^{n}\right)$ is precisely the intersection of those primary components $\mathfrak{p}_{i}$ of $I$ for which $\mathfrak{p}_{i}+\mathfrak{a} \neq$ $\mathbb{Z} A$ [12], p. 218. Thus in our case $\bigcap_{n=1}^{\infty}\left(k \mathbb{Z} A+a^{n}\right)=k \mathbb{Z} A$ and each $\alpha_{i}$ is divisible by $k$. It follows that $t$ is divisible by $k$, so $x$ has a $k$-th root in $G$, and the criterion of Theorem A assures us this root is in $M$.

Incidentally, it can be shown that $M \cap \operatorname{ker} \bar{\eta}_{n}$, which is easily seen to be $M^{\prime} \cap \mathfrak{a}^{n} T$, is $\gamma_{n+1}(M)$. Thus we have a representation, resembling the Magnus representation, of $M / \gamma_{n+1}(M)$, a free group in the variety of groups metabelian and of nilpotency class at most $n$, in the matrix group $G_{n}$.

The following example shows that the hypothesis $x \in M^{\prime}$ cannot be omitted 
from Lemma 4. Let $M$ be a free metabelian group of rank 2 freely generated by $x_{1}$ and $x_{2}$. Every element of $M$ can be written uniquely in the form $x_{1}^{k} x_{2}^{l}\left[x_{1}, x_{2}\right]^{\alpha}$ where $k, l$ are integers and $\alpha \in \mathbb{Z} A$; here $\left[x_{1}, x_{2}\right]^{\alpha}$ denotes the action (defined in section 1) of $\alpha \in \mathbb{Z} A$ on the element $\left[x_{1}, x_{2}\right]$ of $M^{\prime}$. Now consider the element

$$
x=x_{1}^{6}\left[x_{1}, x_{2}\right]^{\left(1+a_{1}\right)\left(1+a_{1}+a_{1}^{2}\right)} .
$$

If $x$ were a proper power, then it would be a power of an element of the form $y\left[x_{1}, x_{2}\right]^{\alpha}$ where $y$ is a power of $x_{1}$ and we would have

$$
x=\left(y\left[x_{1}, x_{2}\right]^{\alpha}\right)^{k}=y^{k}\left[x_{1}, x_{2}\right]^{\alpha\left(1+y M^{\prime}+\cdots+\left(y M^{\prime}\right)^{k-1}\right)} .
$$

But $\left(1+a_{1}\right)\left(1+a_{1}+a_{1}^{2}\right)$ is not divisible by $1+a_{1}+\cdots+a_{1}^{5}, 1+a_{1}^{2}+a_{1}^{4}$ or $1+a_{1}^{3}$, so $x$ is not a 6 th, 3rd, or 2 nd power and hence not a proper power. However,

and

$$
1+a_{1}+\cdots+a_{1}^{5}=\left(a_{1}^{2}+a_{1}+1\right)\left(a_{1}+1\right)\left(a_{1}^{2}-a_{1}+1\right),
$$

Thus,

$$
\left(a_{1}^{2}-a_{1}+1\right) \mathbb{Z} A+\mathfrak{a}=\mathbb{Z} A \text {. }
$$

$$
\bigcap_{n=1}^{\infty}\left(\left(1+a_{1}+\cdots+a_{1}^{5}\right) \mathbb{Z} A+a^{n}\right)=\left(a_{1}^{2}+a_{1}+1\right)\left(a_{1}+1\right) \mathbb{Z} A .
$$

Therefore $\left(a_{1}^{2}+a_{1}+1\right)\left(a_{1}+1\right)$ is divisible by $\left(1+a_{1}+\cdots+a_{1}^{5}\right)$ modulo $\mathfrak{a}^{n}$ for all $n$, so for each $n$ there exists $\alpha_{n} \in \mathbb{Z} A$ and $\beta_{n} \in \mathfrak{a}^{n}$ such that

Hence

$$
\left(a_{1}^{2}+a_{1}+1\right)\left(a_{1}+1\right)=\alpha_{n}\left(1+a_{1}+\cdots+a_{1}^{5}\right)+\beta_{n} .
$$

$$
x=x_{1}^{6}\left[x_{1}, x_{2}\right]^{\alpha_{n}\left(1+a_{1}+\cdots+a_{1}^{5}\right)}\left[x_{1}, x_{2}\right]^{\beta_{n}}=\left(x_{1}\left[x_{1}, x_{2}\right]^{\alpha_{n}}\right)^{6}\left[x_{1}, x_{2}\right]^{\beta_{n}} .
$$

A straightforward induction shows that an element of the form $\left[x_{1}, x_{2}\right]^{\beta_{n}}$ where $\beta_{n} \in \mathfrak{a}^{n}$ lies in $\gamma_{n+2}(M)$. Thus $x$ is a 6 th power modulo every term of the lower central series of $M$. We note, however, that Lemma 4 is true for elements of the form $x=\left(\begin{array}{ll}a_{1}^{p} & 0 \\ t & 1\end{array}\right)$ where $p$ is a prime; for then $1+a_{1}+\cdots+a_{1}^{p-1}$ generates a prime ideal in $\mathbb{Z} A$ and

$$
\bigcap_{n=1}^{\infty}\left(\left(1+a_{1}+\cdots+a_{1}^{p-1}\right) \mathbb{Z} A+\mathfrak{a}^{n}\right)=\left(1+a_{1}+\cdots+a_{1}^{p-1}\right) \mathbb{Z} A .
$$

LEMMA 5 (cf. [4], Lemma 2) Let $N$ be a free group in the variety of metabelian groups of nilpotency class $c$. Let $J$ be the group of automorphisms of $N$ which induce the identity on $N / N^{\prime}$ and let $I$ be the group of inner automorphisms of $N$. Then $J / I$ is torsion-free.

Proof. The proof is identical with that in [4] as soon as one has the following: Suppose $N$ is freely generated by $z_{1}, z_{2}, \cdots$. If $g \in N$ and $\left[g, z_{i}\right]=h_{i}^{k}$ with $h_{i} \in \gamma_{c}(N)$, then $g=h^{k} \bmod \gamma_{c}(N)$ for some $h \in \gamma_{c-1}(N)$. This follows readily 
from the theorem (W. Magnus, see [8], Theorem 36.32) that every element of $N$ can be uniquely expressed as a product of left-normed basic commutators.

THEOREM 2. Let $S$ be a free solvable group, let $J$ be the group of automorphisms of $S$ which induce the identity on $S / S^{\prime}$, and let I be the group of inner automorphisms of $S$. Then $J / I$ is torsion-free.

Proof. Let $l$ be the derived length of $S$. The case $l=1$ is trivial.

We now assume that $l=2$, so that $S=M$. We denote by $h_{n}$ the natural homomorphism of the automorphism group of $M$ into the automorphism group of $M / \gamma_{n}(M)$. For $w$ in any group we denote by $a(w)$ the inner autmorphism induced by $w$.

Now suppose that $\alpha \in J$ and $\alpha^{k} \in I$, say

$$
\alpha^{k}=a(x) .
$$

Then $\left(\alpha h_{3}\right)^{k}=a\left(x \gamma_{3}(M)\right)$; so by Lemma $5 \alpha h_{3}$ is inner, say $\alpha h_{3}=a\left(y \gamma_{3}(M)\right)$. Let

Now,

$$
\beta=\alpha a\left(y^{-1}\right) \text {. }
$$

$$
\beta^{k}=\alpha^{k} a\left(y^{-1}\right)^{\alpha^{k-1}} a\left(y^{-1}\right)^{\alpha^{k-1}} \cdots a\left(y^{-1}\right),
$$

so $\beta^{k}$ is inner since $\alpha^{k}$ is inner and each conjugate of $a\left(y^{-1}\right)$ is inner. Therefore, for some $z \in M$,

$$
\beta^{k}=a(z)
$$

Since $\beta h_{3}$ is the identity mapping of $M / \gamma_{3}(M)$, so is $\beta^{k} h_{3}=a\left(z \gamma_{3}(M)\right)$; therefore $z \gamma_{3}(M)$ is in the center of $M / \gamma_{3}(M)$, i.e., $z \in \gamma_{2}(M)$. The proof now continues as in [4]. For each $n$

$$
\left(\beta h_{n}\right)^{k}=a\left(z \gamma_{n}(M)\right),
$$

so by Lemma $5 \beta h_{n}$ is inner: say $\beta h_{n}=a\left(u_{n} \gamma_{n}(M)\right)$. Then $a\left(u_{n}^{k} \gamma_{n}(M)\right)=a\left(z \gamma_{n}(M)\right)$, whence $a\left(z u_{n}^{-k} \gamma_{n}(M)\right)$ is the identity, which means $z u_{n}^{-k} \gamma_{n}(M)$ is in the center of $M / \gamma_{n}(M)$, which is $\gamma_{n-1}(M) / \gamma_{n}(M)$. Therefore $z u_{n}^{-k} \in \gamma_{n-1}(M)$, and $z$ is a $k$-th power modulo every term of the lower central series of $M$. Because $z \in M^{\prime}$, by Lemma $4 z$ is a $k$-th power, $z=u^{k}$. Thus $\beta^{k}=a\left(u^{k}\right)=a(u)^{k}$. Since $J$ is a group with unique roots [4], Corollary $1, \beta=a(u)$. But since $\beta$ is inner, $\alpha=\beta a(y)$ is also inner.

For $l>2$, we proceed by induction. Let $\theta \in J$ and $\theta^{k} \in I$. Then $\theta$ induces an automorphism of $S / \delta_{t-1}(S)$ which by the induction hypothesis is inner. Thus there is an $x \in S$ such that the automorphism $\eta=\theta a\left(x^{-1}\right)$ induces the identity on $S / \delta_{l-1}(S)$. Now, since $\eta^{k}$ is again inner and induces the identity on $S / \delta_{l-1}(S)$, it must be that $\eta^{k}=a(y)$ with $y \in \delta_{1-1}(S)$. Thus if $\tilde{\eta}$ is the restriction of $\eta$ to the free metabelian group $\delta_{l-2}(S)$, then $\tilde{\eta}$ induces the identity on $\delta_{l-2}(S) / \delta_{l-1}(S)$ and $\tilde{\eta}^{k}$ is still inner. Thus $\tilde{\eta}$ is an inner automorphism of $\delta_{l-2}(S)$, say $\tilde{\eta}=a(z)$ with 
$z \in \delta_{l-2}(S)$. It then follows that $\eta a(z)^{-1}$ fixes $\delta_{l-2}(S)$ pointwise. By Theorem $\mathrm{C}$, $\eta a(z)^{-1}$ is then inner. Thus finally $\theta=\left(\eta a(z)^{-1}\right) a(z) a(x)$ is inner and the theorem is proved.

\section{Free solvable groups as subgroups}

We can now derive some properties of solvable groups with free solvable subgroups of the same derived length.

THEOREM 3. Let $S$ be a non-cyclic free solvable group of derived length $l>1$, and suppose that $S$ is contained in a group $G$ which is torsion-free and solvable of the same length $l$. If $S$ is of finite index in $G$, then $S=G$.

Proof. We first prove the theorem with $l=2$. Then $S$ is our usual free metabelian group $M$. As usual we put $A=M / M^{\prime}$ and we consider $G^{\prime}$ as a $\mathbb{Z} A$-module. Since $M$ is of finite index in $G, M$ contains a subgroup $N$, still of finite index, which is normal in $G$. Let $g \in G^{\prime}$, and let $x$ be in $N$ but not in $M^{\prime}$. Then $[g, x] \in G^{\prime} \cap N$. Since $M^{\prime}$ is a maximal abelian subgroup of $M, G^{\prime} \cap M=M^{\prime}$ and thus $[g, x] \in M^{\prime}$. Let $x M^{\prime}=a$. Then in module notation we have both $k g \in M^{\prime}$ for some integer $k$ and $(a-1) g \in M^{\prime}$. If $t_{1}, t_{2}, \cdots$ are free generators of the Magnus module for $M$ we then have

$$
\begin{array}{rlrl}
k g & =\Sigma \alpha_{i} t_{i} & & \alpha_{i} \in \mathbb{Z} A \\
(a-1) g & =\Sigma \beta_{i} t_{i} & \beta_{i} \in \mathbb{Z} A .
\end{array}
$$

Thus $\Sigma(a-1) \alpha_{i} t_{i}=\Sigma k \beta_{i} t_{i}$ and hence $(a-1) \alpha_{i}=k \beta_{i}$ for all $i$. Since $\mathbb{Z} A$ is a unique factorization domain and $k$ and $(a-1)$ are coprime we find that $k$ divides $a_{i}$ for all $i$, say $\alpha_{i}=k \alpha_{i}^{\prime}$. Thus $k g=\Sigma k \alpha_{i}^{\prime} t_{i}$. Since $\Sigma k \alpha_{i}^{\prime} t_{i}$ represents a group element of $M^{\prime}$, the criterion of Theorem A assures us that $\Sigma \alpha_{i}^{\prime} t_{i}$ also does. Since $G$ is torsionfree, $g=\Sigma \alpha_{i}^{\prime} t_{i}$. Thus $g \in M^{\prime}$ and hence $G^{\prime}=M^{\prime}$.

$M$ is then normal in $G$, and if $h \in G, h$ induces on $M$ an automorphism which leaves $M / M^{\prime}$ invariant. Since some power of $h$ is in $M$, it follows from Theorem 2 that $h$ induces an inner automorphism of $M$. Thus, there exists $u \in M$ with $h u^{-1}$ centralizing $M$. Again for some integer $j,\left(h u^{-1}\right)^{j} \in M$ and hence $\left(h u^{-1}\right)^{j}$ is in the center of $M$. Thus $\left(h u^{-1}\right)^{j}=1$. Since $G$ is torsion-free, $h=u$ and $G=M$ as claimed.

We can now complete the proof with an easy induction. If $l>2$, then since $S^{\prime}$ is a maximal solvable-of-class- $(l-1)$ subgroup of $S, G^{\prime} \cap S=S^{\prime}$ and thus $S^{\prime}$ is of finite index in $G^{\prime}$, and so we have $S^{\prime}=G^{\prime}$. The proof now proceeds as above.

The next theorem follows immediately from the theorem of Smel'kin quoted above.

THEOREM 4. Let $S$ be a free solvable group of derived length $l$ and not cyclic, and suppose that $S$ is a normal subgroup of a group $G$ which is also of derived length $l$. Then $\delta_{l-1}(G)=\delta_{l-1}(S) \times K$ where $K$ centralizes $S$. 
Proof. Let $K$ be a subgroup of $\delta_{t-1}(G)$ maximal with respect to the properties that $g p\left(\delta_{l-1}(S), K\right)=\delta_{l-1}(S) \times K$ and $[S, K]=1$. Let $g \in \delta_{l-1}(G)$. The automorphism $g$ induces on $S$ leaves every element of $\delta_{l-1}(S)$ fixed; therefore by Theorem $C$ this automorphism is an inner automorphism of $S$ induced by an element $h$ of $\delta_{l-1}(S)$. Therefore $g h^{-1}$ centralizes $S$. Let $L=g p\left(g h^{-1}, K\right)$. Then $[S, L]=1$. Further, if $f \in L \cap \delta_{l-1}(S)$, then $f$ is in the center of $S$ and so is 1 . Thus, from the maximality of $K, K=L$. Therefore $g h^{-1} \in K$, so $g \in g p\left(K, \delta_{l-1}(S)\right)$. It follows that $\delta_{l-1}(G)=\delta_{l-1}(S) \times K$.

\section{Some self-normalizing subgroups}

It follows easily from Theorem 4 that a non-abelian free solvable group has no proper normal free subgroups of the same derived length. One can, however, prove more.

THEOREM 5. Let $S$ be a free solvable group of derived length $l>1$ and suppose that $H<S$ is also free of length $l, H$ not cyclic. Then $H$ coincides with its normalizer.

Proof. Assume first that $l=2$ and let $K$ be the normalizer of $H$. Then it can be seen by considering a free nilpotent image of class 2 that $H$ and $K$ coincide modulo $S^{\prime}$. Let $x \in K \cap S^{\prime}$. Then $x$ induces an automorphism of $H$ which fixes $H^{\prime}$ pointwise. Then, by Theorem $\mathrm{C}, x$ induces an inner automorphism of $H$ and hence $x \in H^{\prime}$, for otherwise $H$ would have a non-trivial centralizer. Thus $H=K$. If $l>2$, then, by an obvious induction, $H \cap S^{\prime}=K \cap S^{\prime}$ since $H \cap S^{\prime}=H^{\prime}$ is again free. Further $H=K \bmod S^{\prime}$ as before. So again $H=K$.

In the finitely generated metabelian case we can find another class of selfnormalizing subgroups; we first prove a lemma.

LeMma 6. Let $R$ be a commutative ring with 1 and $T$ a finitely generated $R$ module. Suppose $U$ and $V$ are submodules of $T$ with $U<V<T$ and suppose there is an ideal $I$ of $R$ with the properties

i) $U+I T=T$

ii) $I V<U$.

Then $U=V$.

Proof. Assume that $V \neq U$. Then (see e.g. [9]) there is a maximal ideal $\mathrm{m}$ of $R$ such that the localized modules $U_{m}$ and $V_{m}$ (over the local ring $R_{\mathfrak{m}}$ ) are still distinct. Two cases arise.

1) $I$ is not contained in $\mathrm{m}$. Let $x \in \Lambda \mathfrak{m}$. Then $x$ is a unit in $R_{\mathrm{m}}$, and $V_{\mathrm{m}} x \subset U_{\mathrm{m}}$ implies $V_{\mathrm{m}}=U_{\mathrm{m}}$.

2) $I \subset \mathfrak{m}$. In this case $U_{\mathfrak{m}}+I_{\mathrm{m}} T_{\mathrm{m}}=T_{\mathfrak{m}}$, and the Nakayama Lemma (see e.g. [9]) yields $U_{\mathrm{m}}=T_{\mathrm{m}}$, so that again $U_{\mathrm{m}}=V_{\mathrm{m}}$. 
Our assumption is then untenable and $U=V$ as required.

THEOREM 6. Suppose $M$ is free metabelian of finite rank, and $H<M$ with $H M^{\prime}=M$. Then $H$ coincides with its normalizer.

Proof. Let $K$ be the normalizer of $H$, and let $U$ and $V$ be the $\mathbb{Z} A$-modules generated by the lower left-hand entries of the elements of $H$ and $K$ respectively. $H M^{\prime}=M$ implies that $U+M^{\prime}=T$, and since $M^{\prime} \subset \mathfrak{a} T$,

$$
U+\mathfrak{a} T=T .
$$

Also, $H M^{\prime}=M$ implies that $K=H\left(K \cap M^{\prime}\right)$ and therefore

$$
V=U+\left(K \cap M^{\prime}\right) \text {. }
$$

Since for $g_{1}, g_{2}$ in $M^{\prime}$ and $x$ in $M$ we have $\left[x g_{1}, g_{2}\right]=\left[x, g_{2}\right]$, it follows that

$$
\left[M, K \cap M^{\prime}\right]=\left[H M^{\prime}, K \cap M^{\prime}\right]=\left[H, K \cap M^{\prime}\right],
$$

which is contained in $H \cap M^{\prime}$ and therefore in $U$. But $\left[M, K \cap M^{\prime}\right]=\mathfrak{a}\left(K \cap M^{\prime}\right)$, so

$$
\mathfrak{a} V=\mathfrak{a} U+\left[H, K \cap M^{\prime}\right] \subset U .
$$

The hypotheses of Lemma 6 are then satisfied with $I=\mathfrak{a}$, and hence $U=V$. But then, by Lemma 3, $K=H$ and the theorem is proved.

Theorem 6 is false without the hypothesis of finite rank. Let $M$ be freely generated by $x_{1}, x_{2}, \cdots$ and let

Let

$$
g_{\imath}=\left[x_{i+1}, x_{i+2}\right] \quad i=0,1, \cdots .
$$

and let

$$
y_{i}=x_{i} g_{i} \text { and } z_{i}=\left[y_{i}, g_{0}\right] \quad i=1,2, \cdots,
$$

$$
H=g p\left(y_{1}, y_{2}, \cdots, z_{1}, z_{2}, \cdots\right) .
$$

Since $\left[y_{i}, g_{0}^{-1}\right]=\left[y_{i}, g_{0}\right]^{-1}$ we have $y_{i}^{g_{0}}=y_{i} z_{i}, y_{i}^{g_{0}-1}=y_{i} z_{i}^{-1}, z_{i}^{g_{0}}=z_{i}$, and $z_{i}^{g_{0}^{-1}}=z_{i}$; therefore $g_{0}$ normalizes $H$. Now,

If $g_{0} \in U$, then

$$
U=\bmod \left(t_{1}+g_{1}, t_{2}+g_{2}, \cdots\right)+a g_{0} .
$$

$$
g_{0}=\sum_{i=1}^{n} \rho_{i}\left(t_{i}+g_{i}\right)+\alpha g_{0}
$$

with $\rho_{i} \in \mathbb{Z} A, \alpha \in \mathfrak{a}$. Since $g_{n}=\left(a_{n+2}-1\right) t_{n+1}-\left(a_{n+1}-1\right) t_{n+2}$ is the only term that involves $t_{n+2}$ it follows that $\rho_{i}=0$ for $i=n, n-1, \cdots, 1$ and hence that $\alpha=1$, a contradiction. Thus $g_{0} \notin H$.

Since Theorem 6 is true for finitely generated free metabelian groups, it is also true for all finitely generated metabelian groups. Even further, we have the 
COROLLARY. Let $G$ be a finitely generated solvable group such that all but possibly the last term $\delta_{t-1}(G)$ of the derived series of $G$ are finitely generated. If $H<G$ and $H \delta_{l-1}(G)=G$, then $H$ coincides with its normalizer.

Proof. We use Theorem 6 and the remark above as a basis for an induction on derived length. If $l>2$ and $G$ and $H$ satisfy the hypotheses of the theorem, then $G^{\prime}$ is finitely generated and $G^{\prime}=\left(G^{\prime} \cap H\right) \delta_{l-1}(G)$. If $K$ normalizes $H$, then $K \cap G^{\prime}$ normalizes $H \cap G^{\prime}$ and hence, by induction, $K \cap G^{\prime}=H \cap G^{\prime}$. It follows immediately that $K=H$.

\section{Some finitely generated subgroups}

THeOREM 7. Let $G$ be a finitely generated abelian by polycyclic group, i.e., $G$ has an abelian normal subgroup $N$ such that $G / N$ is polycyclic and $G$ is finitely generated. Let $\left\{H_{i}\right\}$ be an ascending sequence of subgroups of $G$ with $\left[G: H_{i} N\right]<\infty$. Then the sequence is stationary after finitely many steps.

Proof. Since $G / N$ is polycyclic, the sequence $\left\{H_{i} N\right\}$ of subgroups becomes stationary after finitely many steps, so we may assume that $H_{1} N=H_{2} N=\cdots$. Further, since $H_{1} N$ being of finite index in $G$ is again finitely generated abelian by polycyclic, we may assume that $G=H_{1} N$. Let $A=G / N$. Then, since $N$ is abelian, each $H_{i} \cap N$ is normal in $G$, and hence is a $\mathbb{Z} A$-module. Thus since $\mathbb{Z} A$ is noetherian and $N$ is a finitely generated $\mathbb{Z} A$-module [5] we must have, for some $m, H_{m} \cap N=$ $H_{m+1} \cap N=\cdots$. Since we already have $H_{m} N=H_{m+1} N=\cdots$, we must have $H_{m}=H_{m+1}=\cdots$.

COROLlary. With the hypotheses of Theorem 7, if $H<G$ and $H N$ is of finite index in $G$, then $H$ is finitely generated.

Proof. Suppose that $H$ is infinitely generated, say by $z_{1}, \cdots, z_{n}, h_{1}, h_{2}, \cdots$, where $h_{i} \in N$ and the $z_{i}$ generate a subgroup which, modulo $N$, is of finite index in $A$. Then the groups $H_{i}=g p\left(z_{1}, \cdots, z_{n}, h_{1}, \cdots, h_{i}\right)$ form a strictly increasing sequence of groups, a contradiction to the theorem.

\section{References}

[1] S. Bachmuth, 'Automorphisms of free metabelian groups, Trans. Amer. Math. Soc. 118 (1965), 93-104.

[2] G. Baumslag, 'Some subgroup theorems for free b-groups', Trans. Amer. Math. Soc., 108 (1963), 516-525.

[3] G. Baumslag, 'Some theorems on the free groups of certain product varieties', J. of Combinatorial Theory 2 (1967), 77-99.

[4] G. Baumslag, and Tekla Taylor, 'The centre of groups with one defining relator', Math. Annalen 175 (1968), 315-319.

[5] P. Hall, 'Finiteness conditions for soluble groups', Proc. London Math. Soc. (3), 4 (1954), $419-436$. 
[6] I. Kaplansky, Commutative rings, Queen Mary College mathematics notes (Queen Mary College, London, 1966).

[7] W. Magnus, 'On a theorem of Marshall Hall', Ann. of Math. 40, (1939), 764-768.

[8] Hanna Neumann, Varieties of groups (Springer-Vurlag, New York Inc., 1967).

[9] D. G. Northcott, An introduction to homological algebra, (Cambridge University Press, 1960)

[10] A. L. Smel'kin, 'Two notes on free soluble groups', (Russian), Algebra i. Logika Seminar 6 (1967), 95- 109.

[11] J. Stallings, 'On torsion free groups with infinitely many ends', Ann. of Math. (2) 88 (1968), $312-334$.

[12] O. Zariski, and P. Samuel, Commutative algebra, Vol. 1 (Van Nostrand, Princeton, 1968).

Mathematics Department

Syracuse University

Syracuse, N.Y. 13210, U.S.A. 